\title{
Research on Parametric Modeling Technology of Weaving in Micro-Architecture Manufacture
}

\author{
Changzheng Gao ${ }^{1,2, *}$, Bowen $\mathrm{Chen}^{3}$, Yubo $\mathrm{Li}^{2}$, Han Zhang ${ }^{2}$ \\ ${ }^{1}$ School of Public Administration, China University of Geosciences (Wuhan), Wuhan, China \\ ${ }^{2}$ School of Architecture, North China University of Water Resources and Electric Power, Zhengzhou, \\ China \\ ${ }^{3}$ School of Art and Design, North China University of Water Resources and Electric Power, Zhengzhou, \\ China \\ *Corresponding author.
}

\begin{abstract}
With the assistance of parametric technology, micro-architecture design based on weaving is easier to express and apply accurately. This paper analyzes the parametric modeling principle of weaving in micro-architecture design, and studies the parametric modeling technology of weaving motif micro-architecture with the example of microarchitecture shared station design. The conclusion shows that the core parameter model of micro-architecture with weaving motif includes parallel line weaving micro-architecture, gradual weaving micro-architecture and random weaving micro-architecture, each of which has its own corresponding micro-architecture function, and the parameterization technology has an obvious optimization effect on the micro-architecture's envelope shape and manufacturing. Based on this, the combination of micro-architecture and advanced modeling technology is preliminary discussed to promote the design of micro-architecture to be more efficient, ecological and innovative, to promote the upgrading of micro-building manufacturing engineering.
\end{abstract}

Keywords: Micro-architecture, parametric design, weaving theme, intelligent manufacturing, Design and manufacture

\section{Introduction}

The term "micro-architecture" was first used by Charlie Derhoughton to the construction field from the computer field to refer to "light, mobile and ecological future architecture"[1]. The experiments of micro-architecture tend to design themselves as carriers of new technologies and materials, with the development of digital construction, parameterization will be the trend of micro-architecture design[2]. As the motif of design, weaving with unit repetitive texture, which is widely used in micro-architecture design. Weaving has been used in micro-architecture design for a long time. From New Zealand Tree House Restaurant designed by Peter Aixing to KREOD Public Hall designed by ChunQingLi,thanks to parameterization techniques that make previously difficult tasks easy[3]. Therefore, under the trend of parametric design, it is necessary to study the principle of parametric technology with weaving motif, summarize its design paradigm to facilitate the development of micro-architecture to digital construction.

In the past, the research on micro-architecture and weaving mainly focused on the definition, analysis and experiment of its field. Research on the theory and development of micro-architecture. Micro-architecture has the characteristics of light weight, high efficiency and adaptability, as well as the characteristics of delicate, variable and polysemy space. Which make the "living micro space" proposed to solve the housing problem has become a feasible solution to the contradiction between population and space resources and gives new vitality to the nonliving space[4-6]. Combining with new materials and new technologies, the micro-architecture gets its further improvement. The combination of micro-architecture and new materials improves and optimizes its comprehensive performance; the combination of micro-architecture and new technology breaks the closed state of traditional self-

ISSN: 0010-8189

(c) CONVERTER 2020 
contained system and tends to intelligent development[7-8]. The application and development of weaving. Weaving activity originates from the thinking of "knot" formed in people's continuous practice. With the accumulation of experience, the weaving texture is gradually enriched and develops towards diversification. A large number of researches also prove the superiority of weaving structure itself in the application of architecture[9-10] The parametric method has a unique advantage in the design of woven architecture skin, so the application of digital technology in the design of woven architecture skin will be one of the future development directions of architecture[11]. Parametric technology provides a geometric algorithm and physical simulation method for generate weaving strcture, and its feasibility and superiority has been verified[12]. The two basic features of micro-architecture, make it of economic significance, social significance and ecological significance, while the "capacious" and "transparent" formed by weaving make the micro-architecture more light and integrate with the surrounding environment, reflecting the design significance of the micro-architecture. Previous studies have shown that parametric technology provides an effective way for the application and development of weaving. The practical significance of micro-architecture is diverse and extensive and tends to be combined with new technology and new materials. However, the application of micro-architecture is mostly limited to the urban area, and the practical advanced technology mode of micro-architecture design is rarely discussed. Therefore, on the basis of previous relevant research, this paper will summarize the parametric design model under the combination of micro-architecture and weaving texture. Taking micro-architecture design as an example, it will simulate and verify the advantages of parametric technology applied to weaving motif micro-architecture design, provide reference for other micro-architecture parametric design, and expand the application scope of micro-architecture.

\section{Manuscript Preparation}

\subsection{The parametric principle of weaving micro-architecture}

Parametric design is a computer-aided design method based on digital algorithm. Its essence is to find a relationship or rule, organize the main factors that affect the design together, and convert the data information into images by computer technology to get the design results[13]. Grasshopper, a parametric platform used in this paper, is a plug-in tool under rhino modeling software. It has the characteristics of visibility, efficiency, accuracy and expandability. It corresponds to the characteristics of polysemy, miniaturization, refinement and innovation of micro-architecture. Compared with other parametric platform, Grasshopper is more suitable for the parametric design modeling of micro-architecture with weaving motif. Based on the characteristics of weaving motif and the design mode of parameterization technology, the parameterization principle of micro-architecture with weaving motif is explored (Fig.1).

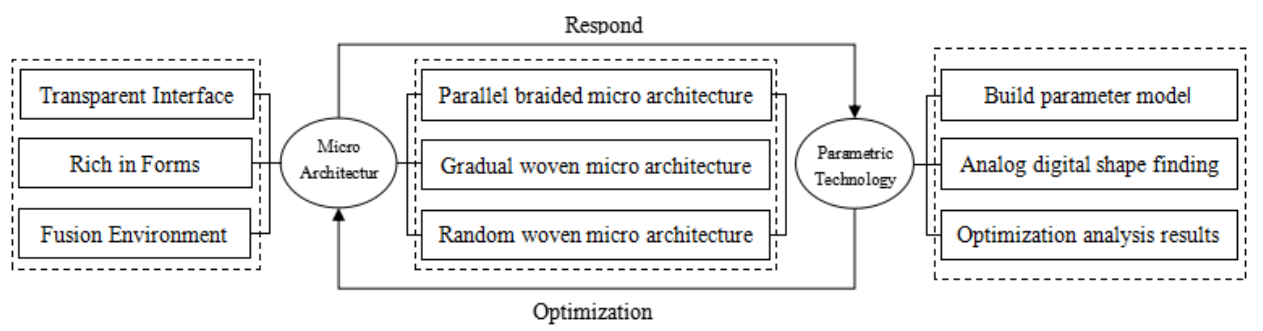

Fig 1: Research framework.

2.1.1 Build parameter model

Building parametric model is the basis of parametric modeling of micro-architecture with weaving motif, and the most essential prototype of weaving motif micro-architecture. It can change itself to generate many forms with different appearances but the same essence. In order to construct the micro-architecture parameter model of weaving motif, it is necessary to analyze the factors that affect its design comprehensively, extract the most core factors for research, find out the logical relationship between design variables, and use the parameterized computer language to express it. By combing the parameter model, the parametric design law of micro-architecture with weaving motif is concluded, which effectively simplifies the design and application steps. As the prototype of the ISSN: 0010-8189

(C) CONVERTER 2020

www.converter-magazine.info 
design module, the parameter model is directly related to the specific design form to get the prototype of the scheme and improve the efficiency of design presentation.

\subsubsection{Analog digital shape finding}

Analog digital shape finding is not only a way of parametric modeling of micro-architecture, but also a necessary step in the whole design process. Based on the corresponding parameter model, the digital shape finding is combined with the specific design scheme to conduct shape generation simulation by the parameterized computer algorithm. The most important feature of the algorithm is that it can be generated automatically according to the computer algorithm language, and the intermediate process can be modified directly without repeating the following steps. On the one hand, digital shape finding has visibility, when the model is generated, the advantage of the algorithm displays instantly, and the human-machine interaction is realized efficiently. On the other hand, digital shape finding is cyclical. When the scheme is refined and modified, the real-time synchronous display of detail adjustment and final effect can be realized, which enriches the design possibility.

\subsubsection{Optimization - Result Analysis}

The optimization model is the adjustment step of the parameterized modeling process of weaving motif. Based on the analysis of the preliminary model, the optimization model optimizes the simulation results of digital shape finding by modifying the parameters. The optimization model specifically includes: Optimize the accuracy of the model, modify the fixed target index, improve the accuracy of the model to provide reference for the subsequent construction; optimize the visual effect, observe the model display results, adjust the corresponding parameter values to optimize the model texture; optimize the ecological performance, and carry out virtual experiments on the reliability of the skin, energy consumption, structure and other factors of the model, which are to provide feedbacks in advance and be adjusted in combination with the deepening consideration of the design[14]. Model optimization under parametric technology, from scheme prototype to scheme deepening, integrates performance objectives into the design optimization of the whole process of the scheme to provide support for design decision.

\subsection{Weaving micro-architecture parameterized model}

Parametric model is a framework for parametric operation. A good parametric model can be used to build logic based on morphology. The micro-architecture parametric model of braided motif is composed of the function and form of micro-architecture as parameters, including various parametric models. From the perspective of modeling technology, micro-architecture braided texture parameter model is the core, which can be arbitrarily combined with other factors (Fig.2). Two basic elements of structure line and structure point are abstracted from the characteristics of braiding, and then Weaving units are formed and combined into various forms. According to the form of Weaving unit, it can be divided into three categories which is parallel weave micro-architecture parameterized model, gradual braided micro-architecture parametric model and random weave micro-architecture parametric model. In Grasshopper, weaving motif is the basic modeling logic of first establishing a Nurbs curve (implied direction of $\mathrm{U}$ and $\mathrm{V}$ direction of grid structure line surface), the weave texture pieces as a whole structural plane, the second by subdividing structural plane is the calculator logic network weaving texture line, finally it is concluded that needs to be woven entity. Following is a brief introduction to the modeling logic of the three core parameter models.

2.2.1 Parallel weave micro-architecture parameterized model

Parallel weave micro-architecture parameterized model is a basic weave texture parameter model, which means that the woven structural lines of micro-architecture skin are parallel to each other, and the geometric units are in the form of regular changes (Fig.2a). Many buildings have been similar design form, such as the roof of expo Hannover in Germany, Louvre Abu Dhabi, The French Pavilion at the 2010 World Expo in Shanghai, Silkworm Cocoon Building in Tokyo. Parallel weave texture is very regular on the surface, without movement, but due to the characteristics of human perspective, it naturally forms changes in walking, the regular arrangement of the line texture for the visual increase of this kind of movement.

The biggest characteristic of parallel line braid texture is regular unit repetition. The main modeling work in ISSN: 0010-8189

(C) CONVERTER 2020 
Grasshopper is to subdivide the structural plane and give it shape by using calculator logic. The following is a brief introduction to the principle of parametric modeling of braid mechanism by taking the braid of hexagonal units as an example. First, Nurs structural planes were built and picked up into Grasshopper, UCount was carried out, and Surface Box was carried out after the braided density was determined in U direction and V direction, and the frame of each three-dimensional braided unit was determined in the structural plane. Then build Polygon in Grasshopper, select the appropriate number of edges, Loft it into a dough, Base it with the thickness of $\mathrm{z}$-axis and Bounding Box to build the required shape of three-dimensional folding unit. Finally, Base, Bounding Box and Surface Box are connected through Box Morph to build corresponding models. The texture thickness of parallel line braid is positively correlated with the Height parameter value of Surface Box.

\subsubsection{Gradual braided micro-building parametric model}

Gradual braided micro-architecture parametric model is a deformed braided texture parametric model, which refers to the random gradient of the braided structure lines of the micro-architecture skin, and the interwoven units are irregular and rhythmic (Figure.2b).For example, "Bird's Nest" stadium, Czech Stork's Nest Farm, Nine Bridge Golf Club and Roof structure of Mumbai International Airport in India have adopted the above design form in the roof structure. The law of gradual braided is not as obvious as that of parallel weave, which forms a whole through seemingly disordered interlacing and achieves a visual harmonious texture.

The generation principle of tapered braided texture is based on the operation mode in Grasshoper, in which random commands can be used to disrupt regular grid points and generate grid lines, and various forms can be generated by setting the parameter value of random interference and adjusting the number of grid lines. The key steps of the parametric modeling principle for such braided mesh generation are as follows. (1) Generate initial regular grid lines. First, a surface whose edges can be divided into multiple UV points is built and picked up in Grasshopper. Then, DvideSurface is carried out to subdivide the surface average UV direction points. An interpoltList of uv points is randomly shifted at Series to generate curves that bend perpendicular to the surface before using an Interpolate wire, then an interpolated grid is formed using the Brep/Plane command.(2) Randomly subtract the grid curve and adjust the density. By adjusting the R-terminal data in List (L) AS list, the generated regular grid lines are randomly deleted in different quantities, and the remaining grid lines are evenly distributed through adjusting the S-terminal data. Then the data is adjusted by repeated operations to achieve a visually harmonious random interleaving grid. (3) Generate woven surface. First, the adjusted grid line structure is merged, then OffsetonSrf grid line offset is carried out and offset data is adjusted by Distance, then Flatten Tree and Graft Tree are used to integrate the data structure, and finally Loft generates the required irregular woven surface. The degree of deformation of graded braided texture lines is positively distributed with the N parameter value of Series, while the number of texture lines is negatively correlated with the Reduction parameter value of Random Reduce.

\subsubsection{Random weave micro-building parametric model}

Random weave micro-architecture parametric model is a refined braided texture parameter model, which refers to the irregular intersection of braided structure lines of the micro-architecture skin, and the interleaved units show a dynamic form of similarity (Figure.2c). For example, the surface structure of National Swimming Center adopts the above design form. The texture of Random weave has no rules to follow on the whole, and its biggest characteristic is that it has no characteristics, so it brings people a kind of disordered visual experience.

The main generation principle of random braiding texture is to use Noronoi3D segmentation to generate abstract braided mesh. This command can be used to generate radial three-dimensional segmentation shapes, then adjust the edges and separate the mesh structure. The key steps of the parametric modeling principle for such braided mesh generation are as follows. (1) Segmentation initial surface. First, the basic surface was established and random structure points were generated from Populate Geometry in Grasshopper. Noronoi3D was used to generate the three-dimensional network structure to be segmented. Then divide the initial surface by Solid Difference command and divide the segmented parts into data by monolithic BREp. Volume and Scale can be combined to Scale and adjust the structure on the basis of box. (2) Extract the required grid part. The grid surface was sorted by CullPattern by making a series of logical calculations of Equality by using the same domain based on Bounds in

ISSN: 0010-8189

(C) CONVERTER 2020

www.converter-magazine.info 
Grasshoper. Then the MeshBrep3 based on Settings is used to separate the grid structure required for modeling. (3) Grid optimization. The separated structural parts were welded by Weaverbird 'sJoinMeshesandWeld and adjusted by Strength, Iterations and Limit in SmoothMeshd, then Weaverbird' sMeshThickena was moved out to thickness, and finally the surface smoothness optimization of Weaverbird 'Scatmull-ClarkSubdivision was carried out to obtain smooth and varied abstract mesh weaving network. With the knitting texture density of the model and Count parameter value are positively correlated, the recommended shape parameters of micro-architecture space within 20 square meters are as follows: the Scale parameter value should be about 1.5, and the Count parameter value of Populate Geometry should be about 200.
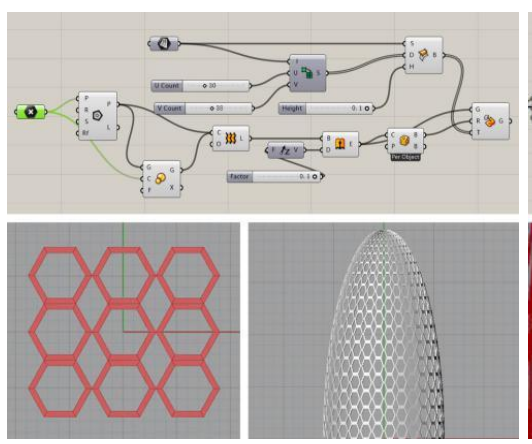

a Parallel line weaving micro-architecture

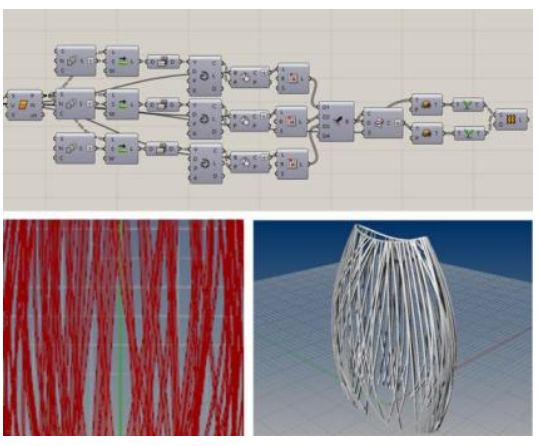

b Gradual weaving micro-architecture Fig 2: Woven micro-architecture parameter model

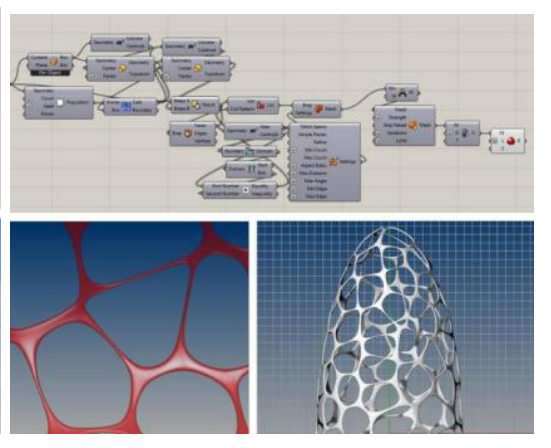

c Random weaving micro-architecture

Through the above research and analysis on braided texture parameter model of core micro-architecture, it can be proved that compared with traditional modeling, braided motif micro-architecture modeling with the assistance of parametric technology has obvious advantages. In traditional modeling, the process of assigning the woven structure to shape according to shape is done manually, which is difficult and prone to errors. By introducing parametric micro-architecture design and quantifying information, different forms can be easily obtained by changing parameters. By using parametric modeling method, the corresponding changes of the structure surface can be completed instantaneously by establishing the structure surface morphology and then compiling the extension components with computing language, which saves a lot of time for manual operation. The accuracy of the structure is guaranteed. After modeling, the data information, Angle positioning and other digital indicators of the specified structure can be quickly obtained by extracting components, providing reliable data support for the later deepening.

\subsection{Parametric modeling and simulation of woven micro-architecture}

Shared rest station is a kind of micro-architecture, which mostly exists in urban landscape area and rural public space. Its functions include landscape, rest and gathering. The space reflects the characteristics of spaciousness, accessibility and transparency of micro-architecture. The design of the shared rest station in this case, covering an area of about 100 square meters, is located in the waterfront landscape park. It is composed of two mountain shaped spaces of different sizes. The design idea comes from "the bright moon rises from the Tianshan Mountain and passes through the vast sea of clouds" to create the scenery of mountain and sea. The parametric modeling of shared rest station integrates weaving texture to generation scheme, apply parameter model of weaving microarchitecture to design initial shape, adjust parameters to get the optimal design model, so as to verify the software parameter model of weaving micro-architecture.

\subsubsection{Fusion parametric scheme generation}

Under the parametric technology, the biggest difference between the weaving pattern micro-architecture design and the traditional design is logic. To generate the parameterized scheme of shared rest station design, the first is to determine the parameter model used according to the appearance, the next is to carry out the detailed design based on the proper parameter model and the synthesis of its use function and form, the final is to generate the prototype

ISSN: 0010-8189

(C) CONVERTER 2020 
of micro-architecture and then to carry out model optimization.

The appearance design of the shared rest station adopts the natural landscape to apply to the modeling, with abstractly using the mountain for the top modeling and the water for the facade skin, and it uses the parameter model of parallel braided micro-architecture. According to the positioning of function and form, the sharing rest station uses the design methods of repetition and contrast to apply to the space and skin. In terms of space design, there are two spaces with different sizes and staggered distribution for landscape leisure and supporting functions. At the intersection of the space, a gray space for the overall connection of the two spaces is set, and a landscape point is set at the center of the gray space and a rest chair is set around the landscape. In the aspect of skin design, the woven texture with permeability is used, of which one part is used as partition to distinguish the space under the eaves from the outdoor space and the other part is used as skin texture.

\subsubsection{Modeling - parametric digital generation}

Digital shape generation modeling is a necessary process of design, which can effectively show the design effect. Based on its parametric model, the digital shape generation of micro-architecture under parametric technology generates the prototype of micro-architecture design with the advantage of computer algorithm.

The parameter model of shared rest station design is parallel braided micro-architecture, which integrates the existing parameter model directly with the spatial shape and deepens and adjusts to generate the prototype of the model in the parametric software (Fig.3). The generation logic of parallel line braided micro-architecture parameter model is to pick up the plane to divide it linearly and form the braided texture of parallel crossing lines. Based on the above brief generation principle, the shared rest station is parameterized and digitally generated. First of all, the basic shape is established, the range of using the parametric model is determined and picked up to the parametric platform in the software. Secondly, in the parametric platform, the parallel braided micro-architecture parameter model and shape range are integrated. Finally, the prototype of the scheme is generated, and the parameterized model is displayed in the software by using the computer algorithm and the shared rest station model is established.

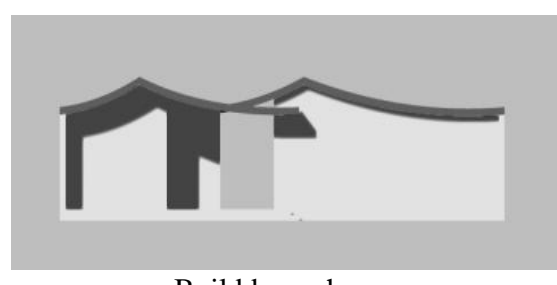

a Build base shape

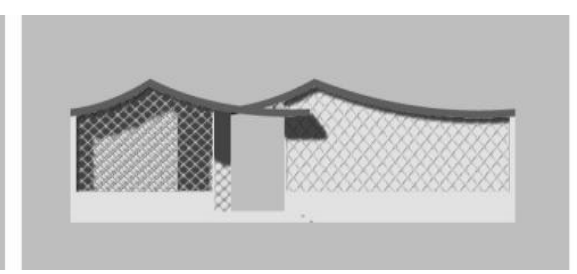

b Fusion parameter model

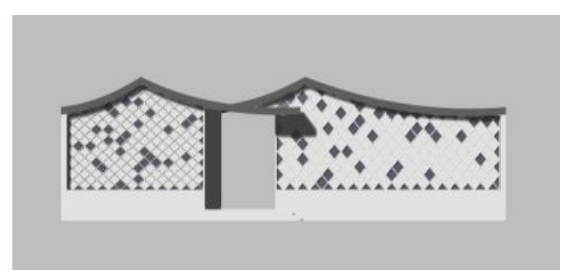

c Generation scheme prototype

Fig 3 Parametric modeling of shared post

\subsubsection{Texture-parameter value regulation and optimization}

Many parameters control commands are used in the generation of weaving texture of shared rest station, and the optimal texture shape cannot be generated at one time due to the influence of model shape and size. Therefore, it is necessary to adjust the parameter values several times to generate the optimal results of the skin weave texture of the shared rest station (Fig.4).

The skin texture of the shared rest station design uses the combination of parallel braided micro parameter model, Random Alix command and Random Reduce command. The parametric model is mainly used to generate parallel braided structural lines. The structural form is mainly determined by the parameter value of the number of $\mathrm{U}$ and $\mathrm{V}$ direction split points. The density of woven structural lines increases with the increase of the parameter value of the split number, the optimal $\mathrm{U}$ and $\mathrm{V}$ parameters in the case are 8 and 41 respectively. The random rotation command is mainly used to rotate the woven structural surface randomly. Its effect is mainly affected by the parameter value of the rotation angle. The random rotation angle of the structural surface increases with the increase of its corresponding parameter value, the best rotation angle parameter value in the case is 22 . The random deletion command is mainly used for random deletion of woven structural surface under the computer algorithm. The effect is mainly affected by the parameter value of the number of deletions. The density of woven structural

ISSN: 0010-8189

(C) CONVERTER 2020

www.converter-magazine.info 
surface decreases with the increase of the parameter value of deletions, the best pruning parameter value in the case is 80 . The most important parameter value is the number of dividing points of woven structure line, which directly affects the parameter value and shaping effect of other two commands.

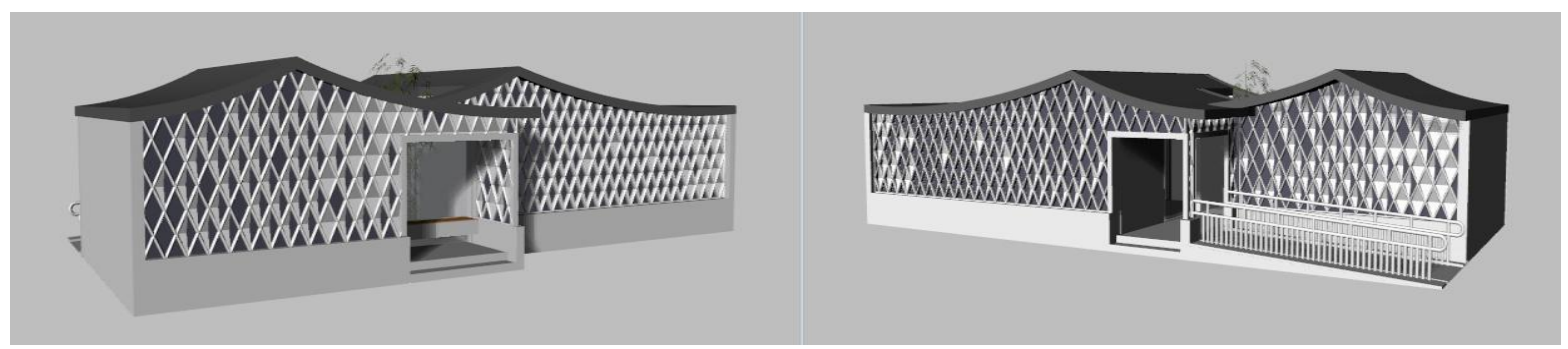

Fig 4: Parameter pptimization results

\section{Conclusion}

The application of micro-building design proves that the comprehensive performance of parametric modeling technology is superior to traditional modeling technology, which can maximize the benefits of technology for design scheme and node manufacturing engineering, and achieve a comprehensive balance of functions, aesthetics, cost and other factors, effectively improving the level of micro-building construction engineering. Through the research on the parametric modeling technology of weaving in micro-architecture design, it is concluded that there are three types of software parameter models of micro-architecture with core weaving motif. According to the morphological and functional characteristics, the corresponding micro-architecture application suggestions are as follows: (1) the parallel line weaving parameter model, with good regularity, can reflect a certain sense of design, but slightly monotonous. It has the least shape limitation, the best effect and the lowest construction difficulty, which is suitable for the micro-architecture with the usage as its main function. (2) The gradual knitting parameter model has a strong sense of natural rhythm and movement, and a strong sense of design. It is suitable for the micro-architecture with visual effect function because of its great limitation, excellent effect and high construction difficulty. (3) The parameter model of random weaving mode has a strong sense of modernity and technology. It is suitable to be used in the micro-architecture with landscape function as the main function.

Under the background of a new round of industrial revolution, digital tools promote the development of a series of man-machine cooperation technologies, which bring the possibility of upgrading to intelligent construction for the construction industry. As an important tool to extend the collaborative scope of designers, parameterization technology achieves the integrated process of micro-architecture design and manufacturing engineering with efficient operation, diverse modeling, accurate simulation parameters and good interaction performance in meeting the basic modeling requirements of micro-architecture design. Microarchitecture design based on parametric technology has obvious advantages and is easy to popularize and popularize. It will be gradually applied from urban space to rural space, and effectively improve the construction of rural architecture from the design end. Micro-architecture is closely combined with new materials and technologies, and more and more widely used parametric technology for a wider range of design manufacturing engineering practice.

\section{Acknowledgements}

This paper was supported by Henan higher education teaching reform research and practice project (2019SJGLX090) and Henan higher education teaching reform research and practice project (Postgraduate Education) 2019SJGLX044Y.

References

[1] R. Horden, P. Richardson, "Micro-architecture: Lightweight, Mobile and Ecological Buildings for the Future," Thames \& Hudson, 2008.

[2] F. Yuan, J.J. Zhou, C. Yan, "Digital craftsman: The future of architecture with human- computer

ISSN: 0010-8189

(C) CONVERTER 2020

www.converter-magazine.info 
collaboration,” Architectural Journal, vol. 04, pp. 1-8, 2019.

[3] D. Harris, S. Harris, "Digital Design and Computer Architecture," Margan Kaufmannn, 2012.

[4] J. Prouve, "Maison Demontable 8x8 Demountable House Hardcover," Galerie Patrick Seguin, 2014.

[5] Beibei Yuanhai, L. Wei, "Research on The Development Trend of "Micro- Architecture". Architecture \& Culture, vol. 10, pp. 45-46, 2013.

[6] J.J. Xi, "Small-scale Public Transportable and Pre-fabricated Buildings: Evaluating Their Functional Performance," Routledge, 2018.

[7] C.Z. Gao, Y.M. Zhang, "A Study on the Application of The Etfe Film Material in "Microarchitectures",’ UPB Scientific Bulletin, Series B: Chemistry and Materials Science, vol. 79, no. 4, pp. 131-142, 2017.

[8] K. Seonwook, M.Y. Pyo, "Construction and Design Manual: Mobile Architecture," Dom Publishers, 2012.

[9] A.C. Corbin, A. Kececi, F. Boussu, et al., "Engineering Design and Mechanical Property Characterisation of 3D Warp Interlock Woven Fabrics," Appl Compos Mater, vol. 25, pp. 811-822, 2018.

[10] S.B. Yan, X. Zeng, A. Long, "Experimental assessment of the mechanical behaviour of 3d woven composite t- joints," Composites Part B: Engineering, vol. 154, pp. 108-113, 2018.

[11] S. Bechert, J. Knippers, O. Kreig, A. Menges, T. Schiwinn, D. Sonntag, "Textile Fabrication Techniques for Timber Shells - Elastic Bending of Custom-Laminated Veneer for Segmented Shell Construction Systems," Advances in Architectural Geometry, 2016.

[12] W.X. Huang, D. Yan, P. Luo, X.L. Li, "Digital Design and Construction of a Weaving Structure," 8th International Conference on Fibre-Reinforced Polymer (FRP) Composites in Civil Engineering (CICE 2016), pp. 1436- 1441, 2016.

[13] W.G. Xu, L. Ning, “Algorithm and Diagram The Digital Diagram from Bio-forms,” Time Architecture. vol. 005, pp. 34-39, 2016.

[14] B. Zhang, "Research on Parametric Regional Architecture Design Based on Ecological Perspective," Proceedings of 2017 4th International Conference on Education, Management and Computing Technology (ICEMCT 2017), pp. 906- 912, 2017. 